INPLASY

PROTOCOL

To cite: Deng et al. Efficacy and safety of moxibustion in the treatment of cancer-related fatigue: a protocol for systematic review and metaanalysis. Inplasy protocol 202110072. doi: 10.37766/inplasy2021.1.0072

Received: 19 January 2021

Published: 19 January 2021

Corresponding author: Gen Deng

1084053154@qq.com

Author Affiliation: Jiangxi University of Traditional Chinese Medicine

Support: Jiangxi Province.

Review Stage at time of this submission: The review has not yet started.

Conflicts of interest: None.

\section{Efficacy and safety of moxibustion in the treatment of cancer-related fatigue: a protocol for systematic review and meta-analysis}

\author{
Deng, G1; Huang, XB2; Tu, MF3; Cheng, QH4; Qiu, Q5; Li, PL6; \\ Pan, ZF?.
}

Review question / Objective: How about the efficacy and safety of moxibustion in the treatment of cancer-related fatigue.

Condition being studied: Cancer-related fatigue (CRF), is a common symptom of cancer patients caused by the interaction of multiple factors, which runs through the whole process of tumor genesis, development, treatment and prognosis. CRF is different from the fatigue after daily body fatigue. It has no obvious relief or relief after rest or sleep, and exists for a long time in the relevant treatment and rehabilitation process. Clinically, CRF is usually treated with western medicine, but the efficacy is short, the recurrence rate is high, the toxic and side effects of drugs are large, and even the adverse reactions of these drugs aggravate the degree of cancer-related fatigue of patients instead. CRF is not only a hot topic in current research, but also an urgent problem in improving the quality of life and prolonging the survival time of cancer patients in clinical practice. In recent years, there have been more and more clinical reports on moxibustion therapy for CRF. However, there is still a lack of systematic evaluation on the efficacy and safety of moxibustion therapy for CRF in clinical practice.

INPLASY registration number: This protocol was registered with the International Platform of Registered Systematic Review and Meta-Analysis Protocols (INPLASY) on 19 January 2021 and was last updated on 19 January 2021 (registration number INPLASY202110072).

\section{INTRODUCTION}

Review question / Objective: How about the efficacy and safety of moxibustion in the treatment of cancer-related fatigue.
Rationale: A systematic review and metaanalysis of clinical randomized controlled studies on moxibustion for cancer-related 
fatigue following the rules of evidencebased medicine.

Condition being studied: Cancer-related fatigue (CRF), is a common symptom of cancer patients caused by the interaction of multiple factors, which runs through the whole process of tumor genesis, development, treatment and prognosis. CRF is different from the fatigue after daily body fatigue. It has no obvious relief or relief after rest or sleep, and exists for a long time in the relevant treatment and rehabilitation process. Clinically, CRF is usually treated with western medicine, but the efficacy is short, the recurrence rate is high, the toxic and side effects of drugs are large, and even the adverse reactions of these drugs aggravate the degree of cancer-related fatigue of patients instead. CRF is not only a hot topic in current research, but also an urgent problem in improving the quality of life and prolonging the survival time of cancer patients in clinical practice. In recent years, there have been more and more clinical reports on moxibustion therapy for CRF. However, there is still a lack of systematic evaluation on the efficacy and safety of moxibustion therapy for CRF in clinical practice.

\section{METHODS}

Search strategy: We will searching Eight electronic databases, including PubMed, Embase, Web of Science, Cochrane Library, the China National Knowledge Infrastructure, Chinese Science and Technology Periodical Database, Wanfang Database, and Chinese Biomedical Literature Database. We will search above electronic databases from the beginning to January 2021, without any language restriction.

Participant or population: There are clear and recognized diagnostic criteria and efficacy criteria, and all patients are diagnosed as CRF, regardless of gender, age and origin of the case.

Intervention: Moxibustion therapy will include all therapies using any type of moxibustion, such as indirect moxibustion, direct moxibustion, heat-sensitive moxibustion, and so on. Mixed therapies based on moxibustion will also be included.Moxibustion therapy, or mixed therapies based on moxibustion will also be include.

Comparator: The control group will receive one of the following treatment methods: conventional pharma-cological therapy, no treatment, and placebo.

Study designs to be included: Clinical randomized controlled trials (RCTs) containing moxibustion for CRF will be included, with no limitation of language and publication status.

Eligibility criteria: Reported in chinese and English, and meet the "PICOS", will be considered for inclusion in this overview.

Information sources: Eight electronic databases will be searched, including PubMed, Embase, Web of Science, Cochrane Library, the China National Knowledge Infrastructure(CNKI), Chinese Science and Technology Periodical Database(VIP), Wanfang Database(WF), and Chinese Biomedical Literature Database(CBM). We will search above electronic databases from the beginning to January 2021, without any language restriction.

Main outcome(s): Clinical efficacy, including total effective rate or cure rate, clinical symptom integral, and recurrence rate will be accepted as the primary outcomes.

Additional outcome(s): The fatigue scale score, quality of life improvement rate will be used as secondary outcomes.

Data management: The two researchers independently read the title and abstract of the literature we obtained, read the full text of the trials that might meet the inclusion criteria to determine whether the inclusion criteria were truly met, and discussed the conflicting literatures or let the third researcher decide whether to include them. Two researchers independently extracted 
data from the included studies, including study design, intervention measures and methods, measurement indicators, results, methodological contents such as hidden grouping and blind method, etc., and a third evaluator checked the consistency of the data. If the required information is incomplete, we will contact the original author for the required data.

Quality assessment / Risk of bias analysis: Two evaluators independently select the literature according to the inclusion and exclusion criteria and cross-check. In case of disagreement, a third evaluator will assist in the decision. The extracted data included the first author, year of publication, number of patients, age, gender, intervention measures, outcome indicators, etc. The Jadad scale to evaluate quality into literature, including: random sequence (right 2 points, 1 points not clear, inappropriate 0 ), distribution, hidden (right 2 points, 1 points not clear, inappropriate 0 ), blinded (right 2 points, 1 points not clear, inappropriate 0 ), lost to follow-up and exit (describe 1 points, not describe 0 ); $0-3$ is classified as low quality and 4-7 as high quality.

Strategy of data synthesis: Meta analysis will be performed using Rev Man5.3.0 software. The odds ratio (OR) and its $95 \%$ Confidence Interval $(\mathrm{Cl})$ will be used as the counting data, while the weighted mean difference (WMD) and its $95 \% \mathrm{Cl}$ will be used as the measurement data.

Subgroup analysis: If the necessary data are available, subgroup analysis will be carried out according to different factors based on the type of CRF, treatment cycle, each moxibustion treatment time, and the type of intervention in the control group.

Sensibility analysis: The purpose of sensitivity analysis is to determine the sources and confounding factors of heterogeneity. If the trial data is sufficient, low or high quality studies will be excluded one by one for sensitivity analysis.
Country(ies) involved: China.

Keywords: moxibustion; cancer-related fatigue; protocol; systematic review and meta analysis.

Contributions of each author:

Author 1 - Gen Deng - The author drafted the manuscript.

Email: 1084053154@qq.com

Author 2 - Xianbao Huang - The author provided statistical expertise.

Email: 515495321@qq.com

Author 3 - Minfang Tu - The author contributed to the development of the selection criteria, and the risk of bias assessment strategy.

Email: 842415798@qq.com

Author 4 - Qinghong Cheng - The author read, provided feedback and approved the final manuscript.

Email: 1208223929@qq.com

Author 5 - Qi Qiu - The author provided statistical expertise.

Email: 997596777@qq.com

Author 6 - Peiling Li - The author contributed to the development of the selection criteria, and the risk of bias assessment strategy.

Email: 532348560@qq.com

Author 7 - Zefeng Pan - The author read, provided feedback and approved the final manuscript.

Email: 862518245@qq.com

Language: No limitation of language. 